\title{
Energetic aspects of glucose metabolism in a pyruvate-dehydrogenase-negative mutant of Saccharomyces cerevisiae
}

\author{
Jack T. Pronk, ${ }^{1}$ Thibaut J. Wenzel, ${ }^{2}$ Marijke A. H. Luttik, ${ }^{1}$ Carolina C. M. \\ Klaassen, ${ }^{1}$ W. Alexander Scheffers, ${ }^{1}$ H. Yde Steensma ${ }^{1,2}$ and Johannes $P$. \\ van Dijken ${ }^{1}$
}

Author for correspondence: Jack T. Pronk. Tel: +31 15 782387. Fax: +3115782355.

\footnotetext{
1 Department of Microbiology and Enzymology, Kluyver Laboratory of Biotechnology, Delft University of Technology, Julianalaan 67, 2628 BC Delft, The Netherlands

2 Department of Molecular and Cellular Biology, Clusius Laboratory, Leiden University,

Wassenaarseweg 64, 2333

AL Leiden, The

Netherlands
}

\begin{abstract}
Saccharomyces cerevisiae T23C (pda1: : Tn5b/e) is an isogenic gene replacement mutant of the wild-type strain $S$. cerevisiae T23D. The mutation causes a complete loss of pyruvate dehydrogenase activity. Pyruvate metabolism in this pyruvate-dehydrogenase-negative ( $P$ dh ) strain was investigated in aerobic glucose-limited chemostat cultures, grown at a dilution rate of $0.10 \mathrm{~h}^{-1}$, and compared with the metabolism in the isogenic wild-type strain. Under these conditions, growth of the Pdh strain was fully respiratory. Enzyme activities in cell-free extracts indicated that the enzymes pyruvate decarboxylase, acetaldehyde dehydrogenase and acetyl-coenzyme $A$ (acetyl-CoA) synthetase could provide a functional bypass of the pyruvate dehydrogenase complex. Since this metabolic sequence involves ATP hydrolysis in the acetyl-CoA synthetase reaction, a negative effect of the pda1::Tn5ble mutation on the growth efficiency was anticipated. Indeed, the biomass yield of the Pdh strain [0.44 $\mathrm{g}$ biomass ( $\mathrm{g}$ glucose) $)^{-1}$ ] was significantly lower than that of wild-type $S$. cerevisiae $\left[0.52 \mathrm{~g}\right.$ biomass ( $\mathrm{g}$ glucose) $\left.{ }^{-1}\right]$. The effect of the mutation on biomass yield could be quantitatively explained in terms of a lower ATP yield from glucose catabolism and an increased ATP requirement for the synthesis of acetyl-CoA used in anabolism. Control experiments showed that the pda1: :Tn5b/e mutation did not affect biomass yield in ethanol-limited chemostat cultures. The results support the view that, during aerobic glucoselimited growth of $S$. cerevisiae at low growth rates, the pyruvate dehydrogenase complex accounts for the major part of the pyruvate flux. Moreover, it is concluded that hydrolysis of pyrophosphate formed in the acetyl-CoA synthetase reaction does not contribute significantly to energy transduction in this yeast. Respiratory-deficient cells did not contribute to glucose metabolism in the chemostat cultures and were probably formed upon plating.
\end{abstract}

Keywords: glucose metabolism, Sacharomyces cerevisiae, pyruvate dehydrogenase, PDA1 gene, pyruvate decarboxylase

\section{INTRODUCTION}

Pyruvate is a key intermediate in sugar metabolism. In Saccharomyces cerevisiae (baker's yeast), pyruvate is located at a branching point between fermentative and respiratory sugar metabolism (Fig. 1). Under anaerobic and oxygenlimited growth conditions, pyruvate is converted to acetaldehyde by pyruvate decarboxylase (EC 4.1.1.1).
Acetaldehyde subsequently serves as an electron acceptor to reoxidize NADH formed in glycolysis. Peculiarly, fermentative metabolism also occurs in S. cerevisiae under fully aerobic conditions at high rates of sugar metabolism (i.e. at high growth rates or in the presence of excess sugar). This phenomenon, called the Crabtree effect, has been attributed to an apparent limited respiratory capacity (Petrik et al., 1983). In S. cerevisiae, completely respiratory 


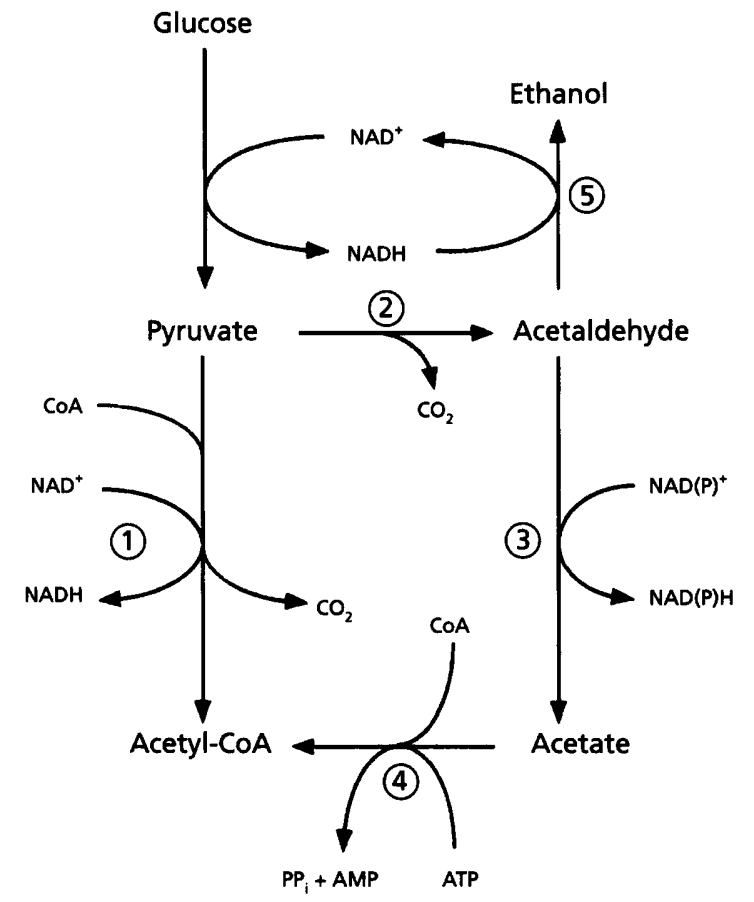

Fig. 1. Enzymes of pyruvate catabolism in $S$. cerevisiae. Numbered reactions are catalysed by the following enzymes: 1, pyruvate dehydrogenase complex; 2 , pyruvate decarboxylase; 3, acetaldehyde dehydrogenase; 4, acetyl-CoA synthetase; 5, alcohol dehydrogenase.

catabolism of sugars only occurs at relatively low growth rates under sugar-limited growth conditions (Petrik et al., 1983; van Urk et al., 1989).

During respiratory growth of $S$. cerevisiae on sugars, pyruvate is converted to acetyl-coenzyme A (acetyl-CoA), which can subsequently be oxidized in the tricarboxylic acid cycle. Formation of acetyl-CoA can occur via two mechanisms (Holzer \& Goedde, 1957; Fig. 1). Oxidative decarboxylation of pyruvate by the mitochondrial pyruvate dehydrogenase (EC 1.2.4.1) complex directly yields acetyl-CoA. Alternatively, the pyruvate dehydrogenase complex can be bypassed by the concerted action of the enzymes pyruvate decarboxylase, acetaldehyde dehydrogenase (EC 1.2.1.4 or EC 1.2.1.5) and acetyl-CoA synthetase (EC 6.2.1.1).

Isolated mitochondria as well as purified pyruvate dehydrogenase complex from $S$. cerevisiae exhibit a higher affinity for pyruvate than the cytosolic enzyme pyruvate decarboxylase (Holzer \& Goedde, 1957; Kresze \& Ronft, 1981 ; van Urk et al., 1989; Postma et al., 1989). In view of its higher affinity for pyruvate, it is assumed that during respiratory, glucose-limited growth of $S$. cerevisiae, pyruvate metabolism occurs predominantly via the pyruvate dehydrogenase complex (Petrik et al., 1983; van Urk et al., 1989). In contrast, at high sugar concentrations (e.g. in batch cultures), intracellular pyruvate concentrations will be high and pyruvate metabolism will also occur via pyruvate decarboxylase. Indeed, pyruvatedecarboxylase-negative distuption mutants of $S$. cerevisiae exhibit very low growth rates in glucose-grown batch cultures (Hohmann \& Cederberg, 1990).

Quantitative determination of pyruvate dehydrogenase activities in cell-free extracts is complicated by the instability of this large multi-enzyme complex and by interference from pyruvate decarboxylase. For this reason, data on the activity of the pyruvate dehydrogenase complex in $S$. cerevisiae as a function of growth conditions are not available. An alternative approach to investigate the role of the pyruvate dehydrogenase complex in sugar metabolism is to study growth of pyruvatedehydrogenase-negative mutants. Recently the $P D A 1$ gene, encoding the E1 $\alpha$ subunit of the $S$. cerevisiae pyruvate dehydrogenase complex, has been cloned (Steensma et al., 1990) and isogenic gene replacement mutants have been constructed using the dominant marker Tn5ble. These pda: :Tn5ble mutants completely lack pyruvate dehydrogenase activity (Wenzel et al., 1992).

In the present study, growth characteristics of wild-type S. cerevisiae during glucose-limited, respiratory growth are compared to those of an isogenic pda1::Tn5ble gene replacement mutant. The aim of the experimental work was to determine whether the enzymes pyruvate decarboxylase, acetaldehyde dehydrogenase and acetyl-CoA synthetase can provide a functional bypass for the pyruvate dehydrogenase complex during aerobic, glucose-limited growth. A model predicting the consequences of the absence of pyruvate dehydrogenase activity on growth efficiency is proposed and experimentally tested.

\section{METHODS}

Yeast strains and maintenance. Saccharomyces cerevisiae T23D (Wenzel et al., 1992) is a homozygous diploid, homothallic wildtype strain, derived from the heterozygous strain $S$. cerevisiae CBS 8066. S. cerevisiae T23C (pda1: : Tn5ble; Wenzel et al., 1992) is isogenic to strain T23D, with the exception of the replacement of the complete $P D A 1$ open reading frame by a yeast expression cassette containing the prokaryotic Tn5ble gene (Wenzel et al., 1992). This mutation confers resistance to the antibiotic phleomycin and completely abolishes pyruvate dehydrogenase activity (Wenzel et al., 1992). Both strains were stored as frozen stock cultures with $20 \%(\mathrm{v} / \mathrm{v})$ glycerol at $-80^{\circ} \mathrm{C}$. Subcultures of these frozen stocks on YPD agar [Difco yeast extract, $2 \%$ $(\mathrm{w} / \mathrm{v})$; Difco peptone, $1 \%(\mathrm{w} / \mathrm{v})$; glucose, $2 \%(\mathrm{w} / \mathrm{v})$; and Difco agar, $2 \%(\mathrm{w} / \mathrm{v})$ ] slants were stored at $4{ }^{\circ} \mathrm{C}$ for no longer than 2 months.

Growth conditions and medium composition. Aerobic chemostat cultivation was performed at $30^{\circ} \mathrm{C}$ in laboratory fermenters (Applikon), at a stirring speed of 800 r.p.m. The working volume of the cultures was kept at 1.0 litre by a peristaltic effluent pump coupled to an Applikon level controller. This setup ensured that under all growth conditions biomass concentrations in samples taken directly from the cultures differed by less than $1 \%$ from biomass concentrations in samples taken from the effluent line. An airflow of $1.51 \mathrm{~min}^{-1}$ through the cultures was maintained using a Brooks 5876 mass flow controller. The dissolved-oxygen concentration was above $50 \%$ air saturation. The culture $\mathrm{pH}$ was maintained at $5 \cdot 0$ by addition of $2.0 \mathrm{M} \mathrm{KOH}$, controlled by an Applikon ADI 1020 bioprocessor. 
The mineral medium, supplemented with vitamins, was prepared according to Verduyn et al. (1992). Vitamins were filtersterilized and added after heat sterilization of the mineral medium. Glucose was sterilized separately at $110^{\circ} \mathrm{C}$. The concentration of ethanol or glucose in the reservoir medium was $5 \mathrm{~g} \mathrm{l}^{-1}$. Cultures were checked for the occurrence of oscillations (Parelukar et al., 1986; Sonnleitner, 1991) by continuous registration of the dissolved-oxygen concentration and $\mathrm{CO}_{2}$ production. All data presented refer to steady states without detectable oscillations.

Control of culture purity and homogeneity. The purity of chemostat cultures was routinely checked by phase contrast microscopy at $1000 \times$ magnification, and by plating of culture samples on YPD agar plates. Stability of the strain carrying the pda 1: : Tn 5ble mutation was checked by replica-plating on YPD agar plates containing $7 \cdot 5 \mu \mathrm{g}$ phleomycin $\mathrm{ml}^{-1}$. To investigate if respiratory-deficient cells were present in the chemostat cultures, appropriate dilutions of culture samples were plated on YPD agar. After $48 \mathrm{~h}$ incubation at $30^{\circ} \mathrm{C}$, colonies were streaked on YPE [Difco yeast extract, $2 \%(\mathrm{w} / \mathrm{v})$; Difco peptone, $1 \%(\mathrm{w} / \mathrm{v})$; ethanol, $2 \%(\mathrm{v} / \mathrm{v})$; and Difco agar, $2 \%$ $(w / v)]$ and YPD plates. Colonies growing on YPD, but not on YPE plates were scored as respiratory deficient.

Determination of culture dry weight. Dry weights of washed culture samples were determined using $0.45 \mu \mathrm{m}$ membrane filters and a microwave oven as described by Postma et al. (1989). Parallel samples varied by less than $1 \%$.

Gas analysis and carbon recovery. Carbon recoveries were calculated from the production of biomass and $\mathrm{CO}_{2}$. Production of $\mathrm{CO}_{2}$ by the cultures was determined and calculated according to van Urk et al. (1988). The carbon content of biomass was assumed to be $45 \%$ of the dry weight (Verduyn et al., 1990).

Analysis of metabolites. Organic acids in culture supernatants were determined by HPLC on an Aminex HPX-87 H column $(300 \times 7.8 \mathrm{~mm}, \mathrm{Bio}-\mathrm{Rad})$ at $30^{\circ} \mathrm{C}$. The column was eluted with $0.5 \mathrm{~g} \mathrm{H}_{2} \mathrm{SO}_{4} \mathrm{I}^{-1}$ at a flow rate of $0.6 \mathrm{ml} \mathrm{min} \mathrm{m}^{-1}$. Detection was by means of a Waters $441 \mathrm{UV}$ detector set at $214 \mathrm{~nm}$, coupled to a Waters 741 data module. Peak areas were linearly proportional to concentrations. The detection limits $(20 \mu \mathrm{l}$ samples) for pyruvate and acetate were below 50 and $200 \mu \mathrm{M}$, respectively. Glucose in reservoir media was assayed with the Boehringer UV kit (no. 716 251); glycerol in media and cultures was assayed with Boehringer kit no. 148270 (detection limit ca. $10 \mu \mathrm{M}$ ). Ethanol was assayed colorimetrically with an alcohol oxidase/ peroxidase kit (Leeds Biochemicals; detection limit ca. $100 \mu \mathrm{M}$ ).

Protein determination. Protein concentrations in cell-free extracts were estimated by the Lowry method. The protein content of culture samples was assayed by a modified biuret method (Verduyn et al., 1990). In both assays, bovine serum albumin (BSA; fatty-acid-free, Sigma) was used as a standard. BSA concentrations in standard solutions were determined with a Hitachi spectrophotometer at $280 \mathrm{~nm}$, assuming an extinction coefficient of $0.661 \mathrm{l} \mathrm{g}^{-1} \mathrm{~cm}^{-1}$ (Wetlaufer, 1962).

Preparation of cell-free extracts. Samples of steady-state chemostat cultures were harvested by centrifugation, washed twice with $10 \mathrm{mM}$ potassium phosphate buffer ( $\mathrm{pH} \mathrm{7.5)} \mathrm{con-}$ taining $2 \mathrm{mM}$ EDTA, concentrated to a final concentration of $10-15 \mathrm{mg}$ dry wt $\mathrm{ml}^{-1}$, and stored at $-20^{\circ} \mathrm{C}$ in $5 \mathrm{ml}$ aliquots. Before preparation of extracts, the samples were thawed at room temperature, washed, and resuspended in ice-cold $100 \mathrm{mM}$ potassium phosphate buffer ( $\mathrm{pH} 7.5)$ containing $1 \mathrm{mM}$ dithiothreitol and $2 \mathrm{mM} \mathrm{MgCl}$. Extracts were prepared by sonication with $0.1 \mathrm{~mm}$ diameter glass beads at $0^{\circ} \mathrm{C}$ for $2 \mathrm{~min}$ at $0.5 \mathrm{~min}$ intervals, using an MSE $150 \mathrm{~W}$ sonicator. Unbroken cells and debris were removed by centrifugation at $75000 \mathrm{~g}(20 \mathrm{~min}$ at $4{ }^{\circ} \mathrm{C}$ ). The clear supernatant was used as the cell-free extract.

Enzyme assays. Activity of the pyruvate dehydrogenase complex was measured as pyruvate-dependent consumption of coenzyme A by partially purified mitochondria, as described by Wenzel et al. (1992). Other enzymes were assayed in cell-free extracts, immediately after the extracts had been prepared. Reaction rates were proportional to the amount of extract added to the assays, which were performed at $30^{\circ} \mathrm{C}$. Pyruvate decarboxylase (EC 4.1.1.1), alcohol dehydrogenase (EC 1.1.1.1, assayed with either $100 \mathrm{mM}$ ethanol or $25 \mathrm{mM}$ pentanol as the substrate), NAD- and NADP-dependent acetaldehyde dehydrogenase (EC 1.2.1.5 and EC 1.2.1.4, respectively), acetyl-CoA synthetase (EC 6.2.1.1) and glucose6-phosphate dehydrogenase (EC 1.1.1.49) were assayed according to Postma et al. (1989). Citrate synthase (EC 4.1.3.7) was assayed according to Srere (1969) and isocitrate lyase (EC 4.1.3.1) according to Dixon \& Kornberg (1959). In all enzyme assays, 1 unit is defined as the amount of enzyme catalysing the conversion of $1 \mu \mathrm{mol}$ substrate $\mathrm{min}^{-1}$. Specific activities are given as $\mathrm{U}$ (mg protein $)^{-\mathbf{1}}$.

Electron microscopy. For electron microscopy, cells were fixed and stained as described by Visser et al. (1990).

\section{RESULTS}

\section{Prediction of the effect of the pda1::Tn5ble mutation on growth efficiency}

Theoretically, conversion of pyruvate to acetyl-CoA in a $\mathrm{Pdh}^{-}$mutant of $S$. cerevisiae can occur by the concerted action of the enzymes pyruvate decarboxylase, acetaldehyde dehydrogenase and acetyl-CoA synthetase (Holzer \& Goedde, 1957; Fig. 1). If hydrolysis of pyrophosphate cannot be coupled to energy conservation, the equivalent of two molecules of ATP (when hydrolysed to ADP and inorganic phosphate) is used in the acetyl-CoA synthetase reaction. In contrast, direct oxidative decarboxylation of pyruvate to acetyl-CoA by the pyruvate dehydrogenase complex does not require ATP hydrolysis (Fig. 1). It is generally assumed that in wild-type $S$. cerevisiae grown under glucose limitation, the pyruvate dehydrogenase complex is primarily responsible for pyruvate catabolism (Petrik et al., 1983; van Urk et al., 1989). For a quantitative estimation of the effect of the pda1: :Tn5ble mutation on growth efficiency during aerobic, glucose-limited growth, the assumption was made that in wild-type $S$. cerevisiae conversion of pyruvate to acetyl-CoA occurs solely via the pyruvate dehydrogenase complex.

The use of the pyruvate decarboxylase bypass in $\mathrm{Pdh}^{-}$ S. cerevisiae and the resulting increased ATP requirement for acetyl-CoA synthesis will cause a decrease of the net ATP yield from aerobic glucose catabolism. Furthermore, because acetyl-CoA is an important 'building block' for biosynthesis, the ATP requirement for anabolism will increase. Both factors will negatively influence the growth efficiency [ $\mathrm{g}$ biomass ( $\mathrm{g}$ glucose consumed) ${ }^{-1}$ ].

Catabolic and anabolic fluxes of glucose and ATP in wildtype $S$. cerevisiae, grown in aerobic, glucose-limited chemostat cultures at a dilution rate of $0 \cdot 10 \mathrm{~h}^{-1}$, are shown in Fig. 2(a). For the formation of $100 \mathrm{~g} \mathrm{~S}$. cerevisiae 


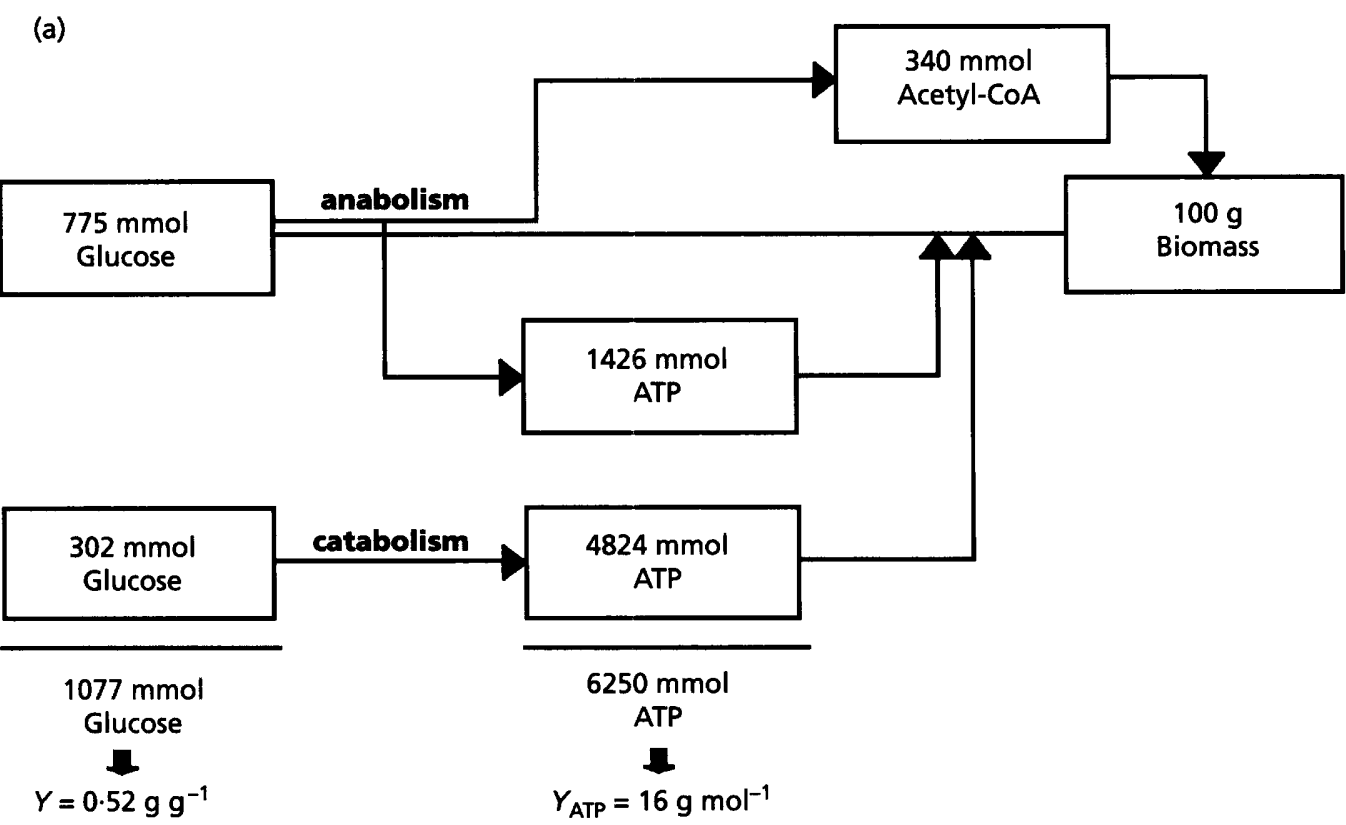

(b)

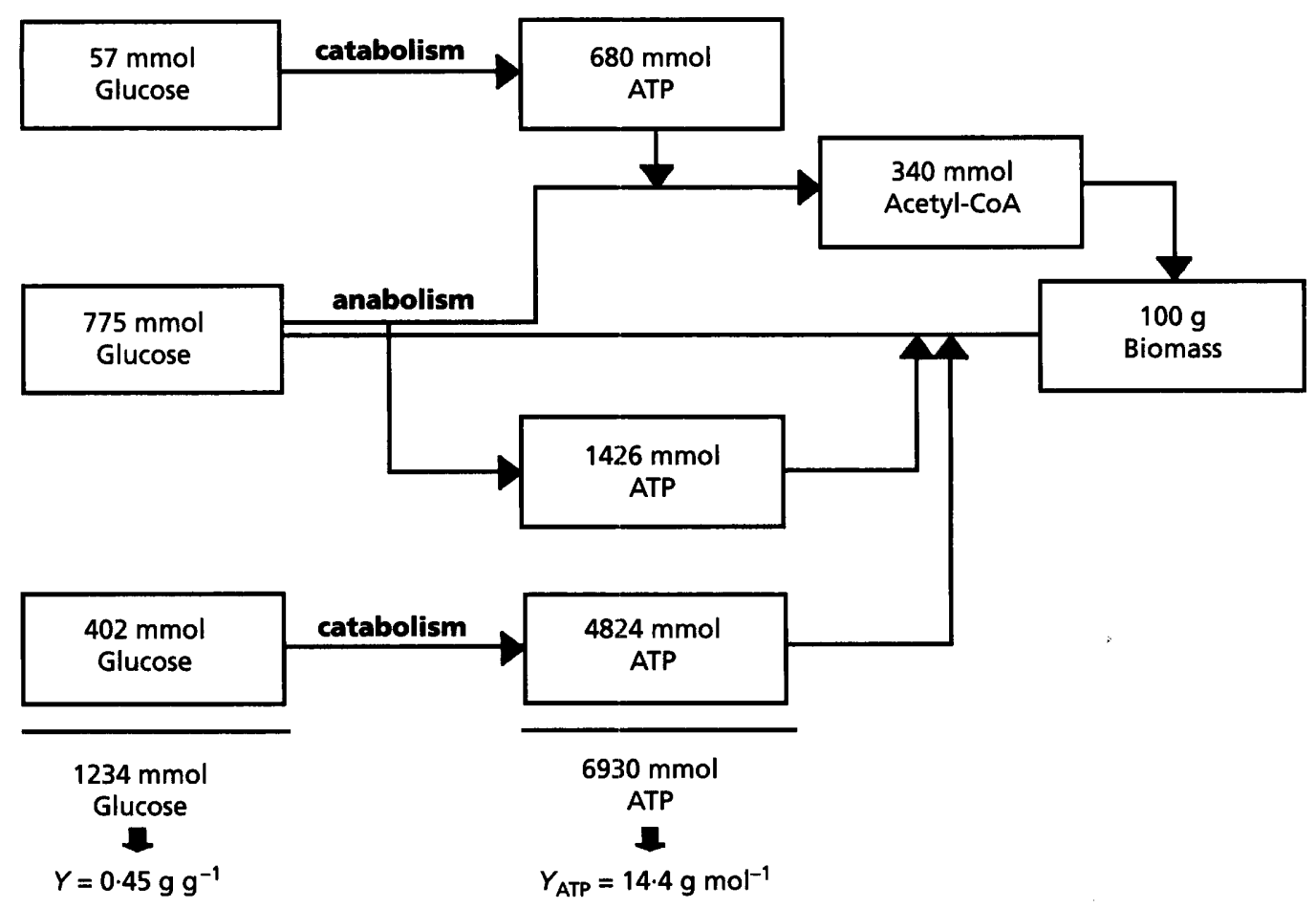

Fig. 2. Schematic representation of (a) metabolic fluxes of glucose and ATP in wild-type S. cerevisiae, grown aerobically in glucose-limited chemostat cultures and (b) predicted fluxes in a $\mathrm{Pdh}^{-}$strain using the pyruvate decarboxylase bypass. It has been assumed that conversion of pyruvate to acetyl-CoA in wild-type $S$. cerevisiae occurs solely via the pyruvate dehydrogenase complex, and that the $\mathrm{Pdh}^{-}$mutant uses the pyruvate decarboxylase bypass for this purpose. The $1426 \mathrm{mmol}$ ATP which appear to be generated during anabolism are derived from the oxidation of reduced cofactors formed during biosynthetic reactions. Data on fluxes of glucose and ATP in wild-type S. cerevisiae are from Verduyn et al. (1991). 
Table 1. Estimation of acetyl-CoA requirement for synthesis of $S$. cerevisiae biomass

The acetyl-CoA requirement for synthesis of the major biopolymers was calculated from Oura (1972). The protein content of $S$. cerevisiae strains $\mathrm{T} 23 \mathrm{D}$ and $\mathrm{T} 23 \mathrm{C}$ was determined experimentally (Table 2). Data on carbohydrate, nucleic acid and lipid contents of yeast biomass are from Oura (1972) and Verduyn et al. (1990, 1991).

\begin{tabular}{|lccc|}
\hline Polymer & $\begin{array}{c}\text { g polymer } \\
\mathbf{( 1 0 0} \mathbf{g} \\
\text { biomass) }\end{array}$ & $\begin{array}{c}\text { mmol } \\
\text { acetyl-CoA } \\
\text { (g polymer) }^{-1}\end{array}$ & $\begin{array}{c}\text { mmol } \\
\text { acetyl-CoA } \\
(\mathbf{1 0 0} \mathbf{~ g} \\
\text { biomass) }^{-1}\end{array}$ \\
\hline Carbohydrate & 39 & 0 & 0 \\
Protein & 40 & $4 \cdot 1$ & 164 \\
Nucleic acid & 7 & 0 & 0 \\
Lipid & 7 & $25 \cdot 1$ & 176 \\
$\quad$ Total... & & & 340 \\
\hline
\end{tabular}

biomass, $0.775 \mathrm{~mol}$ glucose is used in anabolism (Verduyn et al., 1990). With a $Y_{\text {ATP }}$ of $16 \mathrm{~g}$ biomass (mol ATP) $)^{-1}$ (Verduyn et al., 1990), the total ATP requirement for the formation of $100 \mathrm{~g}$ biomass is $6.25 \mathrm{~mol}$ ATP. Part of this requirement is met by oxidation of reduced cofactors formed during anabolism. The remainder is provided by complete catabolism of glucose to $\mathrm{CO}_{2}$ (Fig. 2a). Complete respiratory catabolism of $1 \mathrm{~mol}$ glucose to $\mathrm{CO}_{2}$ yields $4 \mathrm{~mol}$ ATP by substrate-level phosphorylation and $12 \mathrm{~mol}$ of reduced cofactors (NADH and FADH). Assuming an effective $\mathrm{P} / \mathrm{O}$ ratio of 1 (Verduyn $e t$ al., 1991), the total ATP yield from glucose catabolism will be $16 \mathrm{~mol} \mathrm{~mol}^{-1}$.

In a $\mathrm{Pdh}^{-}$mutant using the pyruvate decarboxylase bypass, glucose catabolism yields only $12 \mathrm{~mol}$ ATP (mol glucose) $^{-1}$, because the equivalent of $4 \mathrm{~mol} \mathrm{ATP}$ (mol glucose) $^{-1}$ is used in the acetyl-CoA synthetase reaction. Consequently, the amount of glucose that has to be catabolized to provide ATP for biosynthesis will be $33 \%$ higher than in wild-type S. cerevisiae (Fig. 2b).

To calculate the expected effect of the absence of pyruvate dehydrogenase activity on the synthesis of acetyl-CoA used in anabolism, it was necessary to estimate the amount of acetyl-CoA required for biosynthesis. Based on the work of Oura (1972) it could be calculated that in the formation of $100 \mathrm{~g}$ S. cerevisiae biomass, $340 \mathrm{mmol}$ acetylCoA are used for synthesis of fatty acids and amino acids (Table 1). In the $\mathrm{Pdh}^{-}$mutant, synthesis of acetyl-CoA used in biosynthesis requires $2 \mathrm{~mol} \mathrm{ATP}$ (mol acetyl$(\mathrm{CoA})^{-1}$. This results in an additional ATP requirement of $0.68 \mathrm{~mol}$ for the synthesis of $100 \mathrm{~g}$ biomass (Fig. 2b).

From the model presented in Fig. 2 (a, b), it can be predicted that in the $\mathrm{Pdh}^{-}$mutant, redirection of pyruvate metabolism via the pyruvate decarboxylase bypass will lead to a significant decrease of the growth efficiency. This should be reflected both in a reduction of the biomass yield on glucose and in a decrease of $Y_{\mathrm{ATP}}$. It is not possible to directly measure $Y_{\mathrm{ATP}}$ in aerobic cultures.
However, the magnitude of the predicted decrease of the biomass yield $[0.52 \mathrm{~g} \text { biomass ( } \mathrm{g} \text { glucose })^{-1}$ in wild-type S. cerevisiae versus $0 \cdot 45 \mathrm{~g} \mathrm{~g}^{-1}$ in a $\mathrm{Pdh}^{-}$strain] made it possible to verify the model by measuring growth yields in glucose-limited chemostat cultures.

\section{Growth in ethanol- and glucose-limited chemostat cultures}

The pyruvate dehydrogenase complex is not known to be involved in ethanol metabolism. Therefore, ethanollimited chemostat cultures of wild-type and $\mathrm{Pdh}^{-}$ $S$. cerevisiae were used as a control for possible pleiotropic effects of the pda1::Tn5ble mutation. With both strains, substrate carbon was quantitatively recovered as biomass and $\mathrm{CO}_{2}$ (data not shown). Neither the growth yield on ethanol nor the protein content of the biomass differed significantly between the two strains (Table 2). Furthermore, no significant changes were observed in the activities of a number of key enzyme activities in cell-free extracts (Table 3 ). Even after continuous cultivation for over 50 generations, all cells of the $\mathrm{Pdh}^{-}$strain retained the phleomycin resistance marker (data not shown).

In glucose-limited chemostat cultures of wild-type and $\mathrm{Pdh}^{-}$S. cerevisiae, substrate carbon could be quantitatively recovered as $\mathrm{CO}_{2}$ and biomass also (carbon recoveries in all chemostat cultures were between $97 \%$ and $103 \%$ ). No detectable amounts of ethanol, glycerol or acetate were found in the culture supernatants by HPLC analysis. Low concentrations $(<200 \mu \mathrm{M})$ of a number of organic acids (mainly TCA cycle intermediates) were detected in cultures of both wild-type and $\mathrm{Pdh}^{-}$cultures. Apparently, the absence of pyruvate dehydrogenase activity did not result in aerobic ethanol formation or excretion of other metabolites. Also with the glucose-limited chemostat cultures, no loss of phleomycin resistance was observed in the strain carrying the pda1: :Tn5ble mutation.

The biomass yield of the homozygous diploid wild-type strain S. cerevisiae T23D on glucose was identical to the growth yield of the heterozygous parent strain $S$. cerevisiae CBS 8066 (Verduyn et al., 1991). The protein contents of the wild-type and $\mathrm{Pdh}^{-}$strain were not significantly different (Table 2). However, the biomass yield of the $\mathrm{Pdh}^{-}$strain was significantly lower $(15.4 \%)$ than that of the wild-type strain (Table 2). It has been reported that in batch cultures grown on glucose, the pda1::Tn5ble mutation results in a partial leucine requirement (Wenzel et al. 1992). Addition of leucine $\left(0 \cdot 2 \mathrm{~g}^{-1}\right)$ to the reservoir medium of glucose-limited chemostat cultures of the $\mathrm{Pdh}^{-}$strain did not significantly affect the biomass yield (data not shown). Quantitatively, the observed difference between the biomass yields of the wild-type and $\mathrm{Pdh}^{-}$ strains was in good agreement with the model presented in Fig. 2.

\section{Enzymology of glucose and pyruvate metabolism}

To establish if the pda1: :Tn5ble mutation, which has previously been shown to cause a complete loss of pyruvate dehydrogenase activity in batch cultures (Wenzel et al., 1992), indeed led to the absence of pyruvate 
Table 2. Growth yields and protein contents of S. cerevisiae T23D (wild-type) and S. cerevisiae T23C ( $p d a 1:: \operatorname{Tn} 5 b / e$ ) in ethanol- and glucose-limited chemostat cultures

Growth conditions: $D=0 \cdot 10 \mathrm{~h}^{-1}, \mathrm{pH} 5 \cdot 0, T=30^{\circ} \mathrm{C}, S_{\mathrm{R}}=$ $5 \mathrm{~g} \mathrm{l}^{-1}$. Data are represented as means \pm SD of samples from at least three independent steady-state chemostat cultures.

\begin{tabular}{|c|c|c|c|c|}
\hline \multirow[t]{2}{*}{$\begin{array}{l}\text { Growth } \\
\text { substrate }\end{array}$} & \multicolumn{2}{|c|}{$\begin{array}{l}\text { S. cerevisiae T23D } \\
\text { (wild-type) }\end{array}$} & \multicolumn{2}{|c|}{$\begin{array}{c}\text { S. cerevisiae T23C } \\
(\not p d a 1:: \operatorname{Tn} 5 b l e)\end{array}$} \\
\hline & $\begin{array}{c}\text { Biomass } \\
\text { yield } \\
\text { (g dry } \\
\left.\text { wt } \mathrm{g}^{-1}\right)\end{array}$ & $\begin{array}{c}\text { Protein } \\
\text { content } \\
(\%)\end{array}$ & $\begin{array}{c}\text { Biomass } \\
\text { yield } \\
(\mathrm{g} \text { dry } \\
\left.\text { wt } \mathrm{g}^{-1}\right)\end{array}$ & $\begin{array}{c}\text { Protein } \\
\text { content } \\
(\%)\end{array}$ \\
\hline Ethanol & $0.59 \pm 0.02$ & $40 \cdot 8 \pm 1 \cdot 2$ & $0.59 \pm 0.01$ & $41 \cdot 4 \pm 1 \cdot 8$ \\
\hline Glucose & $0.52 \pm 0.01$ & $40 \cdot 4 \pm 2 \cdot 3$ & $0 \cdot 44 \pm 0.01$ & $39 \cdot 6 \pm 2 \cdot 2$ \\
\hline
\end{tabular}

dehydrogenase activity in glucose-limited chemostat cultures, consumption of CoA by crude mitochondrial preparations was studied. Indeed, pyruvate-dependent CoA consumption was demonstrated with extracts from wild-type cells grown in glucose-limited chemostat cultures, but not with extracts prepared from $S$. cerevisiae T23C (Fig. 3). This confirmed that, also during growth under substrate-limited conditions, the pda1::Tn5ble mutation led to a complete loss of pyruvate dehydrogenase activity.

Activities of the 'bypass' enzymes pyruvate decarboxylase, acetaldehyde dehydrogenase and acetyl-CoA synthetase in cell-free extracts prepared from glucose-limited chemostat cultures of the $\mathrm{Pdh}^{-}$strain were similar to those observed in cultures of the wild-type strain (Table 3). The specific glucose consumption rate in chemostat cultures of the $\mathrm{Pdh}^{-}$strain (equal to the dilution rate

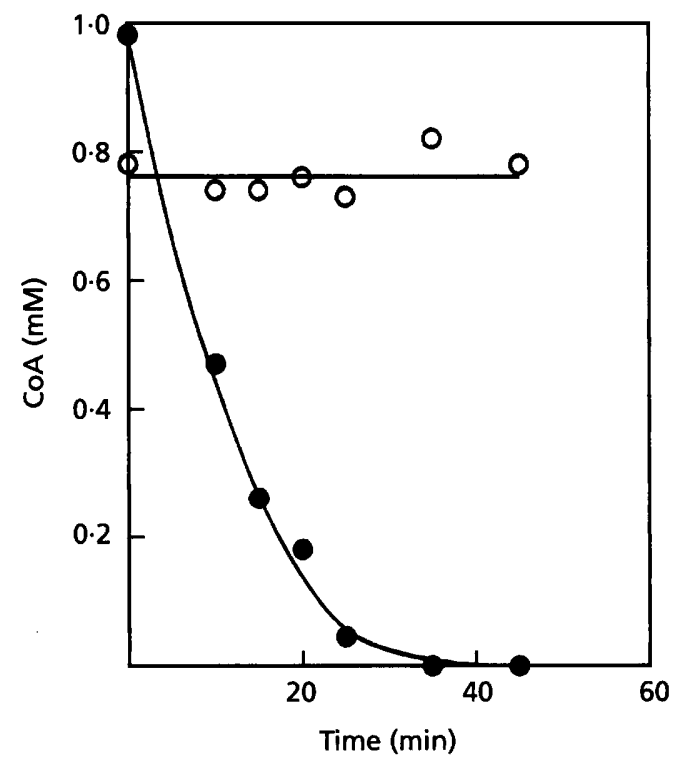

Fig. 3. Pyruvate-dependent consumption of coenzyme $A$ by crude mitochondrial preparations of wild-type and $\mathrm{Pdh}^{-} \mathrm{S}$. cerevisiae, grown in aerobic, glucose-limited chemostat cultures $\left(D=0.1 \mathrm{~h}^{-1}\right.$, $\left.\mathrm{pH} 5, T=30^{\circ} \mathrm{C}\right)$. Symbols: $\mathrm{S}$. cerevisiae T23D (wild-type); O, S. cerevisiae T23C (pda1::Tn5ble). The protein concentration in the assays was $1.3 \mathrm{mg} \mathrm{ml}^{-1}$. The figure shows data from a representative experiment. Absence of activity in extracts of strain T23C was confirmed in five independent experiments.

divided by the molar growth yield on glucose; see Table 2) was $21 \mathrm{nmol}$ glucose $\min ^{-1}(\mathrm{mg} \mathrm{dry} \mathrm{wt})^{-1}$ or $63 \mathrm{nmol}$ $\min ^{-1}$ (mg protein) $)^{-1}$ (assuming a soluble protein content of $33 \%$; Postma et al., 1989). The activities of the bypass enzymes measured in cell-free extracts were all higher than $100 \mathrm{nmol} \mathrm{min}^{-1}$ (mg protein) $)^{-1}$ (Table 3 ) and hence sufficient to account for the in vivo substrate consumption rates.

Table 3. Enzyme activities in cell-free extracts of S. cerevisiae T23D (wild-type) and S. cerevisiae T23C (pda $1:$ :Tn5b/e), grown in aerobic ethanol- and glucose-limited chemostat cultures

Growth conditions: $D=0 \cdot 10 \mathrm{~h}^{-1}, \mathrm{pH} 5 \cdot 0, T=30^{\circ} \mathrm{C}, S_{\mathrm{R}}=5 \mathrm{~g} \mathrm{l}^{-1}$. Enzyme activities are presented as means $\pm \mathrm{sD}$ of extracts prepared from at least three independent steady-state chemostat cultures.

\begin{tabular}{|c|c|c|c|c|}
\hline $\begin{array}{c}\text { Enzyme activity } \\
{\left[\mu \mathrm{mol} \mathrm{min}^{-1}(\mathrm{mg} \text { protein })^{-1}\right]}\end{array}$ & $\begin{array}{c}\text { T23D } \\
\text { (wild-type) } \\
\text { Ethanol }\end{array}$ & $\begin{array}{c}\text { T23C } \\
(p d a 1:: \operatorname{Tn} s b l e) \\
\text { Ethanol }\end{array}$ & $\begin{array}{c}\text { T23D } \\
\text { (wild-type) } \\
\text { Glucose }\end{array}$ & $\begin{array}{c}\text { T23C } \\
(p d a 1:: \text { Tnsble }) \\
\text { Glucose }\end{array}$ \\
\hline Glucose-6-phosphate dehydrogenase & $0 \cdot 24 \pm 0 \cdot 01$ & $0.23 \pm 0.06$ & $0 \cdot 41 \pm 0 \cdot 14$ & $0 \cdot 46 \pm 0 \cdot 11$ \\
\hline Hexokinase & $0.63 \pm 0.09$ & $0 \cdot 73 \pm 0 \cdot 12$ & $1 \cdot 50 \pm 0 \cdot 30$ & $1 \cdot 74 \pm 0 \cdot 17$ \\
\hline Citrate synthase & $0.88 \pm 0.07$ & $0.79 \pm 0.09$ & $0 \cdot 55 \pm 0 \cdot 17$ & $0.85 \pm 0.09$ \\
\hline Pyruvate decarboxylase & $0.75 \pm 0.33$ & $0.47 \pm 0.09$ & $0.67 \pm 0.05$ & $0.78 \pm 0.05$ \\
\hline Acetyl-CoA synthetase & $0 \cdot 90 \pm 0 \cdot 2,1$ & $0 \cdot 50 \pm 0 \cdot 20$ & $0 \cdot 13 \pm 0 \cdot 03$ & $0 \cdot 11 \pm 0 \cdot 04$ \\
\hline Isocitrate lyase & $0 \cdot 28 \pm 0 \cdot 07$ & $0.30 \pm 0.04$ & $0.02 \pm 0.01$ & $<0.003$ \\
\hline Alcohol dehydrogenase (ethanol) & $10 \cdot 7 \pm 2 \cdot 2$ & $13 \cdot 0 \pm 2 \cdot 0$ & $10 \cdot 1 \pm 0 \cdot 5$ & $9 \cdot 84 \pm 0 \cdot 75$ \\
\hline Alcohol dehydrogenase (pentanol) & $7 \cdot 32 \pm 0 \cdot 91$ & $7 \cdot 63 \pm 1 \cdot 84$ & $5 \cdot 65 \pm 0 \cdot 48$ & $3.95 \pm 0.23$ \\
\hline Acetaldehyde dehydrogenase NAD-dependent & $0 \cdot 76 \pm 0 \cdot 11$ & $0.67 \pm 0.08$ & $0.89 \pm 0.07$ & $1 \cdot 31 \pm 0 \cdot 11$ \\
\hline Acetaldehyde dehydrogenase NADP-dependent & $0.27 \pm 0.02$ & $0 \cdot 25 \pm 0 \cdot 04$ & $0 \cdot 31 \pm 0 \cdot 03$ & $0.42 \pm 0.06$ \\
\hline
\end{tabular}


Table 4. Consumption and production of glycerol in aerobic, glucose-limited chemostat cultures of S. cerevisiae T23D (wild-type), S. cerevisiae T23C (pda1: :Tn5ble) and S. cerevisiae T23C-RD (pda1::Tn5ble; spontaneous respiratory-deficient mutant)

Growth conditions: $D=0 \cdot 10 \mathrm{~h}^{-1}, T=30^{\circ} \mathrm{C}, \mathrm{pH} 5, S_{\mathrm{R}}=$ $5.0 \mathrm{~g}_{\text {glucose } \mathrm{l}^{-1} \text {. }}$.

\begin{tabular}{|lccc|}
\hline Strain & $\begin{array}{c}\text { Glycerol in } \\
\text { medium } \\
\mathbf{( m M )}\end{array}$ & $\begin{array}{c}\text { Glycerol in } \\
\text { culture } \\
\mathbf{( m M )}\end{array}$ & $\begin{array}{c}\text { Glycerol } \\
\text { consumed } \\
\mathbf{( m M )}\end{array}$ \\
\hline T23D & $<0.01$ & $<0.01$ & $<0.01$ \\
T23C & $<0.01$ & $<0.01$ & $<0.01$ \\
T23D & 5.18 & 5.01 & 0.17 \\
T23C & 4.60 & 4.51 & 0.09 \\
T23C-RD & $<0.01$ & 5.20 & -5.20 \\
\hline
\end{tabular}

The lower growth yield of the $\mathrm{Pdh}^{-}$strain results in an $18 \%$ increase of the specific glucose consumption rate, as compared to wild-type $S$. cerevisiae. The average activities of hexokinase and citrate synthase appeared to be higher in the $\mathrm{Pdh}^{-}$strain than in wild-type S. cerevisiae (Table 3). However, the experimental variation in the enzyme activities was too large to decide if the increase of the catabolic fluxes in the $\mathrm{Pdh}^{-}$strain is achieved by increased enzyme levels, increased metabolite concentrations, or a combination of these two parameters.

\section{Absence of respiratory-deficient mutants in chemostat cultures}

During growth of $\mathrm{Pdh}^{-}$strains on glucose in batch cultures, a high incidence of respiratory-deficient ('petite') cells was observed (Wenzel et al., 1992). When samples of steady-state, glucose-limited chemostat cultures of the $\mathrm{Pdh}^{-}$mutant were plated on YPD agar, $20-30 \%$ of the colonies exhibited a typical 'petite' phenotype: colonies were small and, upon replica-plating, were unable to grow on YPE plates. This phenomenon was not observed with wild-type $S$. cerevisiae T23D or with ethanol-limited chemostat cultures of the $\mathrm{Pdh}^{-}$ strain. This observation suggested that respiratorydeficient cells were present in the glucose-limited chemostat cultures of the $\mathrm{Pdh}^{-}$strain. Attempts to directly compare numbers of colonies on glucose plates with those on ethanol or acetate plates were not successful, because with wild-type $S$. cerevisiae also, the numbers of colonies on the latter plates were poorly reproducible and consistently lower than on glucose plates (data not shown).

If respiratory-deficient cells contributed to glucose metabolism in chemostat cultures of the $\mathrm{Pdh}^{-}$strain, this would invalidate the model presented in Fig. 2, since the occurrence of alcoholic fermentation in the cultures would also result in a decrease of the growth efficiency. Therefore, the reliability of the replica-plating method in determining the presence of respiratory-deficient cells in the chemostat cultures was investigated by using different methods.

It has been reported that certain fluorescent dyes can be used to detect a mitochondrial membrane potential and, consequently, to discriminate between respiratory-competent and respiratory-deficient cells (Pringle et al., 1989; Skowronek et al., 1990). Attempts to detect respiratorydeficient cells by fluorescence microscopy, using the fluorescent dyes Rhodamine 123, DASPMI [2-(4dimethyl-aminostyryl)- $N$-methylpyridinium iodide] and DIOC $_{6}$ (dihexyloxocarbocyanine iodide) were not successful (data not shown). Even with cultures of wild-type $S$. cerevisiae, large variations were observed in fluorescence among individual cells. With all three dyes, significant fluorescence of organelles was also observed with pure cultures of a $\mathrm{Pdh}^{-}$respiratory-deficient strain. This observation suggests that in respiratory-deficient cells the mitochondria retain a membrane potential. Since direct quantification of the frequency of respiratory-deficient cells in the cultures by fluorescence microscopy was not successful, the presence of fermentation products in the cultures was studied.

A representative respiratory-deficient $\mathrm{Pdh}^{-}$colony (strain T23C-RD) was isolated from a YPD plate. In aerobic, glucose-limited chemostat cultures, the growth yield of this strain $\left.[0.11 \mathrm{~g} \text { (g glucose })^{-1}\right]$ was comparable to that of wild-type $S$. cerevisiae grown in anaerobic, glucoselimited chemostat cultures (Verduyn et al., 1990). Consistent with this, ethanol and glycerol yields $[0.36 \mathrm{~g}$

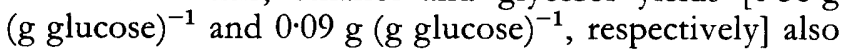
were equal to those in anaerobic cultures of wild-type S.cerevisiae (Verduyn et al., 1990). No ethanol was detectable in culture supernatants of glucose-limited chemostat cultures of $S$. cerevisiae T23C (pda1::Tn5ble) but this might be explained by (co-)metabolism of ethanol by the respiratory-competent part of the population. However, the biomass yield of the respiratory-deficient cells in pure

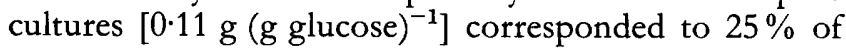
the growth yield of the $\mathrm{Pdh}^{-}$strain in glucose-limited chemostat cultures (Table 2). If it is assumed that the frequency of respiratory-deficient colonies on plates $(20-30 \%)$ accurately reflected the presence of respiratorydeficient cells in the chemostat cultures, this would imply that virtually all glucose supplied to the cultures was initially consumed by respiratory-deficient cells. The respiratory-competent population would then be restricted to the use of ethanol and/or glycerol as carbon sources. Consequently, this should lead to induction of enzymes involved in ethanol metabolism, such as, for instance, isocitrate lyase. This key enzyme of the glyoxylate cycle is induced during growth of wild-type and $\mathrm{Pdh}^{-}$ $S$. cerevisiae on ethanol (Table 3). However, no isocitrate lyase activity could be detected in cell-free extracts of glucose-limited cultures of the $\mathrm{Pdh}^{-}$strain (Table 3), indicating that ethanol is not a major substrate for growth in these cultures. Clearly, this observation is not compatible with the presence of $20-30 \%$ actively growing respiratory-deficient cells in the cultures, as suggested by the plate counts. 


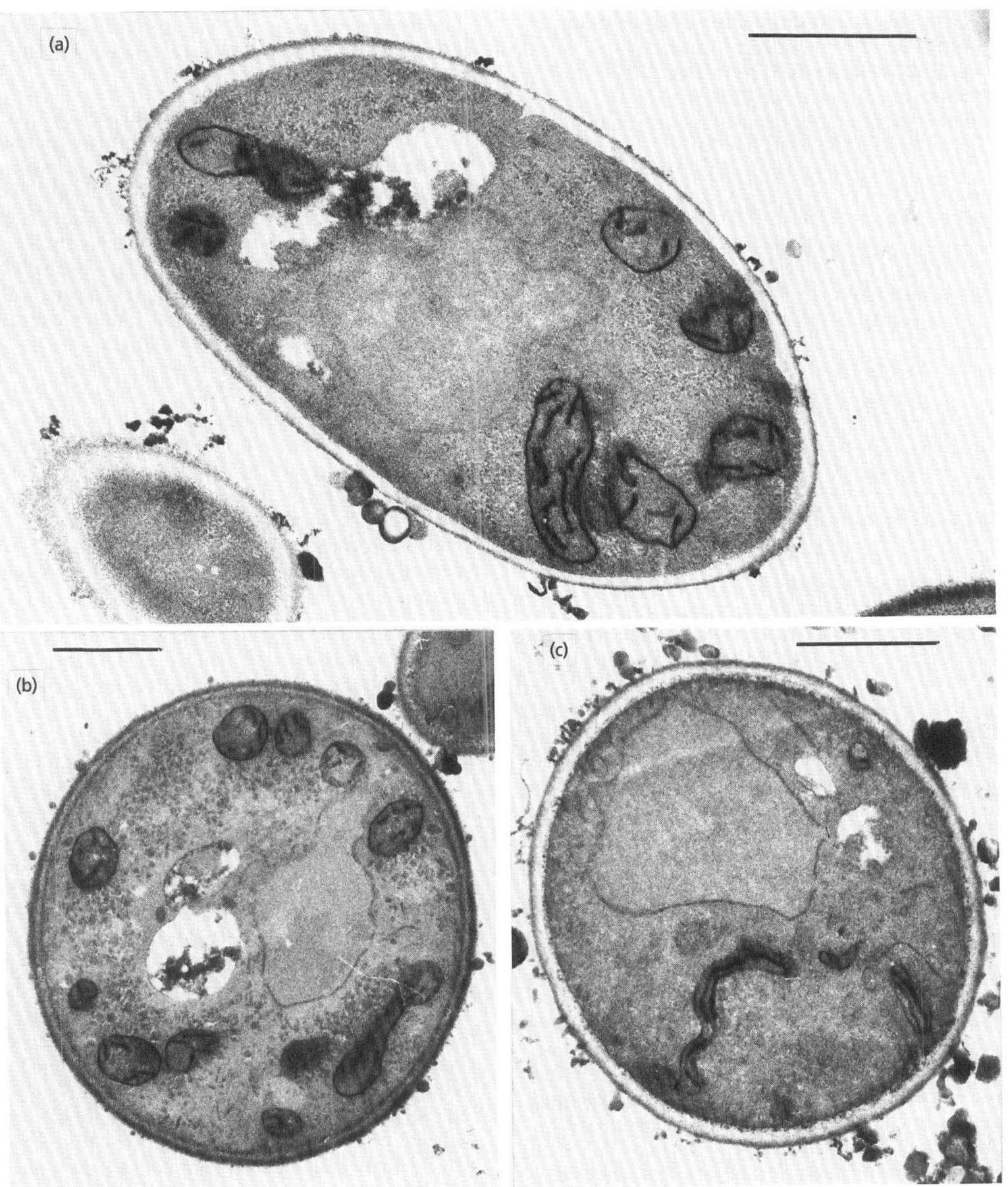

Fig. 4. Electron micrographs of thin sections of cells from glucose-limited chemostat cultures of (a) S. cerevisiae T23D (wild-type); (b) S. cerevisiae T23C (pda1::Tn5b/e); (c) S. cerevisiae T23C-RD (pda1::Tn5ble; spontaneous respiratorydeficient mutant). The marker bars correspond to $1 \mu \mathrm{m}$.

No glycerol was detected in glucose-limited chemostat cultures of wild-type and $\mathrm{Pdh}^{-} S$. cerevisiae. Neither of these strains can use glycerol as a sole source of carbon and energy for growth (data not shown). However, it could not be excluded that small amounts of glycerol produced by respiratory-deficient cells might have been co-metabolized. Therefore, co-metabolism of glycerol was studied by adding low concentrations of glycerol to the reservoir medium of glucose-limited chemostat cultures of both wild-type and $\mathrm{Pdh}^{-} S$. cerevisiae. In all cases, glycerol added to the growth medium could be almost quantitatively recovered from the cultures (Table 4). Apparently, co-metabolism of glycerol does not occur to any significant extent under these growth conditions. The absence of glycerol in glucose-limited chemostat cultures of the $\mathrm{Pdh}^{-}$strain can therefore be interpreted as 
additional proof that respiratory-deficient cells did not contribute significantly to glucose metabolism in these cultures.

Electron microscopy of thin sections of respiratorydeficient cells grown in aerobic, glucose-limited chemostat cultures revealed atypical mitochondrial structures (Fig. 4). In contrast, cells of wild-type and $\mathrm{Pdh}^{-} S$. cerevisiae exhibited a normal mitochondrial morphology with well-defined cristae (Fig. 4). No atypical mitochondrial structures were observed in over 250 sections of cells from glucose-limited chemostat cultures of $\mathrm{Pdh}^{-}$ S.cerevisiae. This observation also indicated that respiratorydeficient cells were not present in the chemostat cultures, but were formed upon plating of $\mathrm{Pdh}^{-}$cells.

\section{DISCUSSION}

\section{Phenotypic characteristics of S. cerevisiae T23C}

Inactivation of the $P D A 1$ gene results in a dramatic increase of the number of respiratory-deficient colonies after plating of samples from glucose-limited cultures onto YPD agar plates. This phenomenon also occurs with batch cultures of $S$. cerevisiae $\mathrm{T} 23 \mathrm{C}$ and other strains in which the PDA1 gene is disrupted (Wenzel et al., 1992). For the interpretation of our experimental data, it was essential to assess whether or not respiratory-deficient cells contributed to glucose metabolism in the glucoselimited chemostat cultures. Both the absence of glycerol in culture supernatants (Table 4) and the absence of isocitrate lyase activity in cell-free extracts of glucoselimited chemostat cultures of $\mathrm{Pdh}^{-}$S. cerevisiae (Table 3) indicate that, if the cultures contain any respiratorydeficient cells, these do not significantly contribute to glucose metabolism. This means that, if present at all in the cultures, the impact of respiratory-deficient cells on metabolic fluxes is comparable with that of dead cells, i.e. their main effect will be an increased growth rate of the remainder of the population. Since the biomass composition of the $\mathrm{Pdh}^{-}$strain was not significantly different from that of wild-type $S$. cerevisiae (Table 2), such an increase in the specific growth rate would not affect the stoichiometric calculations leading to the model presented in Fig. 2.

If the plate counts reflect the presence of respiratorydeficient cells, non-growing in the chemostat cultures, this would imply a very high mutation rate (approximately $0 \cdot 25$ per generation). The absence of cells with an altered mitochondrial morphology in glucose-limited cultures of $\mathrm{Pdh}^{-}$S. cerevisiae (Fig. 4) suggests that respiratorydeficient cells were not present in these cultures. Our current hypothesis is that cells from glucose-limited chemostat cultures of the $\mathrm{Pdh}^{-}$strain contain high activities of a mitochondrial pyruvate transport system, but are unable to metabolize intramitochondrial pyruvate. Transfer to a situation of glucose excess (e.g. on glucosecontaining agar plates) could lead to a rapid influx and accumulation of pyruvate into the mitochondria. If accumulation of pyruvate in the matrix impairs mitochondrial functions, including replication of mito- chondrial DNA, this may explain the observed irreversible loss of respiratory activity.

\section{Energetic implications of the pda1: :Tn5ble mutation}

The experimental data presented in this paper indicate that the enzymes pyruvate decarboxylase, acetaldehyde dehydrogenase and acetyl-CoA synthetase can provide a functional bypass of the pyruvate dehydrogenase complex. The observation that this bypass allows a fully respiratory metabolism demonstrates that redirection of glucose metabolism via pyruvate decarboxylase is not sufficient to trigger alcoholic fermentation. Apparently, the NADH/NAD ratio during aerobic glucose-limited growth is sufficiently low to allow acetaldehyde dehydrogenase to compete effectively with alcohol dehydrogenase. Also, the much higher affinity of acetaldehyde dehydrogenase for acetaldehyde as compared with that of alcohol dehydrogenase (Postma et al., 1989) will favour oxidative metabolism of acetaldehyde.

Quantitatively, the decrease of the biomass yield on glucose caused by the pda1: $: \operatorname{Tn} 5$ ble mutation is in good agreement with the model outlined in Fig. 2. This model was based on a number of assumptions.

(1) In wild-type $S$. cerevisiae grown in aerobic, glucoselimited chemostat cultures, pyruvate catabolism occurs exclusively via the pyruvate dehydrogenase complex. However, the possibility cannot be excluded that in wildtype $S$. cerevisiae also, a fraction of the pyruvate flux is channelled through the pyruvate decarboxylase bypass. This should result in a smaller effect of the pda1: : Tn5ble mutation on biomass yields.

(2) Conversion of ATP to AMP and pyrophosphate in the acetyl-CoA synthetase reaction is equivalent to the hydrolysis of two molecules of ATP to ADP and phosphate. This assumption would not hold if the chemical energy present in pyrophosphate can be conserved. In addition to soluble cytoplasmic pyrophosphatase, the presence of a vacuolar proton-translocating pyrophosphatase has been reported in S. cerevisiae (Lichko \& Okorokov, 1991). Furthermore, it has been suggested that a yeast mitochondrial membrane-bound pyrophosphatase is proton-translocating and may therefore be energy-transducing (Lundin et al., 1992). The relative contribution of these enzymes to the overall rate of pyrophosphate hydrolysis in yeast is unknown.

(3) The effective $\mathrm{P} / \mathrm{O}$ ratio during glucose-limited growth of $S$. cerevisiae is near unity, as calculated by Verduyn et al. (1991). When, in fact, the effective P/O ratio is higher than one, the relative effect of additional ATP expenditure in the pyruvate decarboxylase bypass would be smaller than indicated by Fig. 2 .

If either of the assumptions 1 or 2 are incorrect, the effect of the pda1: :Tn5ble mutation on biomass yield would be smaller than observed. Hence, the observed effect of the pda 1: :Tn5ble mutation on biomass yield confirms that during respiratory, glucose-limited growth of S. cerevisiae, the pyruvate dehydrogenase complex accounts for the 
major part of the pyruvate flux, as has previously been proposed on the basis of kinetic data (Petrik et al., 1983; van Urk et al., 1989). Furthermore, the observed biomass yield of $\mathrm{Pdh}^{-} S$. cerevisiae suggests that hydrolysis of pyrophosphate formed in the acetyl-CoA synthetase reaction does not contribute significantly to energy transduction. The decrease of the biomass yield in the $\mathrm{Pdh}^{-}$strain can be interpreted as further evidence for a rather low efficiency of oxidative phosphorylation (Verduyn et al., 1991) in S. cerevisiae under the experimental conditions.

\section{ACKNOWLEDGEMENTS}

We thank Wilma Batenburg-van der Vegte and Anke de Bruyn for performing electron microscopy and fluorescence microscopy, respectively.

\section{REFERENCES}

Dixon, G. H. \& Kornberg, H. L. (1959). Assay methods for key enzymes of the glyoxylate cycle. Biochem $J 72,3 \mathrm{p}$.

Hohmann, S. \& Cederberg, H. (1990). Autoregulation may control the expression of yeast pyruvate decarboxylase structural genes $P D C 1$ and PDC5. Eur J Biochem 188, 615-621.

Holzer, H. \& Goedde, W. H. (1957). Zwei Wege von Pyruvat zu Acetyl-Coenzym A in Hefe. Biocbem Z 329, 175-191.

Kresze, G. B. \& Ronft, H. (1981). Pyruvate dehydrogenase from baker's yeast. I. Purification and some kinetic and regulatory properties. Eur J Biochem 119, 573-579.

Lichko, L. \& Okorokov, L. A. (1991). Purification and some properties of membrane-bound and soluble pyrophosphatases of yeast vacuoles. Yeast 7, 805-812.

Lundin, M., Deopugari, S. W., Lichko, L., Pereira da Silva, L. \& Baltscheffski, H. (1992). Characterization of a mitochondrial inorganic pyrophosphatase in Saccharomyces cerevisiae. Biochim Biopbys Acta 1098, 217-223.

Oura, E. (1972). The effect of aeration on the growth energetics and biochemical composition of baker's yeast. $\mathrm{PhD}$ thesis, University of Helsinki, Finland.

Parelukar, S. J., Semones, G. B., Rolf, M. J., Lievense, J. C. \& Lim, H. C. (1986). Induction and elimination of oscillations in continuous cultures of Saccharomyces cerevisiae. Biotechnol Bioeng 28, 700-710.

Petrik, M., Käppeli, O. \& Fiechter, A. (1983). An expanded concept for the glucose effect in the yeast Saccharomyces warum: involvement of short- and long-term regulation. J Gen Microbiol 129, 43-49.
Postma, E., Verduyn, C., Scheffers, W. A. \& van Dijken, J.P. (1989). Enzymic analysis of the Crabtree effect in glucose-limited chemostat cultures of Saccharomyces cerevisiae. Appl Environ Microbiol 53, 468-477.

Pringle, J. R., Preston, R. A., Adams, A. E. M., Stearns, T., Drubin, D. G., Haarer, B. K. \& Jones, E. W. (1989). Fluorescence microscopy for yeast. Methods Cell Biol 31, 358-429.

Skowronek, P., Krummeck, G., Haferkamp, O. \& Rodel, G. (1990). Flow cytometry as a tool to discriminate respiratory-competent and respiratory-deficient yeast cells. Curr Genet 18, 265-267.

Sonnleitner, B. (1991). Dynamics of yeast metabolism and regulation. Bioprocess Eng 6, 187-193.

Srere, P. A. (1969). Citrate synthase. Methods Enzymol 13, 3-11.

Steensma, H. Y., Holterman, L., Dekker, I., van Sluis, C. A. \& Wenzel, T. J. (1990). Molecular cloning of the gene for the E1 $\alpha$ subunit of the pyruvate dehydrogenase complex from Saccharomyces cerevisiae. Eur J Biocbem 191, 769-774.

van Urk, H., Mak, P. R., Scheffers, W. A. \& van Dijken, J. P. (1988). Metabolic responses of Saccharomyces cerevisiae CBS 8066 and Candida utilis CBS 621 upon transition from glucose limitation to glucose excess. Yeast 4, 283-291.

van Urk, H., Schipper, D., Breedveld, G. J., Mak, P. R., Scheffers, W. A. \& van Dijken, J. P. (1989). Localization and kinetics of pyruvate-metabolizing enzymes in relation to aerobic alcoholic fermentation in Saccharomyces cerevisiae CBS 8066 and Candida utilis CBS 621. Biochim Biophys Acta 992, 78-86.

Verduyn, C., Postma, E., Scheffers, W. A. \& van Dijken, J. P. (1990). Physiology of Saccharomyces cerevisiae in anaerobic glucoselimited chemostat cultures. J Gen Microbiol 136, 395-403.

Verduyn, C., Stouthamer, A. H., Scheffers, W. A. \& van Dijken, J. P. (1991). A theoretical evaluation of growth yields of yeasts. Antonie Leeuwenboek 59, 49-63.

Verduyn, C., Postma, E., Scheffers, W. A. \& van Dijken, J.P. (1992). Effect of benzoic acid on metabolic fluxes in yeast: a continuous-culture study on the regulation of respiration and alcoholic fermentation. Yeast 8, 501-517.

Visser, W., Scheffers, W. A., Batenburg-van der Vegte, W. H. \& van Dijken, J. P. (1990). Oxygen requirements of yeasts. Appl Environ Microbiol 56, 3785-3792.

Wenzel, T. J., van den Berg, M. A., Visser, W., van den Berg, J. A. \& Steensma, H. Y. (1992). Characterization of mutants lacking the E1 $\alpha$ subunit of the pyruvate dehydrogenase complex from Saccharomyces cerevisiae. Eur J Biochem 209, 697-705.

Wetlaufer, D. B. (1962). Ultraviolet spectra of proteins and amino acids. Adv Protein Chem 17, 303-390.

Received 25 June 1993; revised 13 September 1993; accepted 4 October 1993. 